\title{
Conical refraction as a tool for polarization metrology
}

\author{
Alba Peinado,* Alex Turpin, Angel Lizana, Estefania Fernández, Jordi Mompart, and Juan Campos \\ Departament de Física, Universitat Autònoma de Barcelona, Bellaterra E-08193, Spain \\ ${ }^{*}$ Corresponding author: alba.peinado@uab.es \\ Received June 19, 2013; revised September 5, 2013; accepted September 6, 2013; \\ posted September 9, 2013 (Doc. ID 192625); published October 9, 2013

\begin{abstract}
A method for polarization metrology based on the conical refraction (CR) phenomenon, occurring in biaxial crystals, is reported. CR transforms an input Gaussian beam into a light ring whose intensity distribution is linked to the incoming polarization. We present the design of a division-of-amplitude complete polarimeter composed of two biaxial crystals, whose measurement principle is based on the CR phenomenon. This design corresponds to a static polarimeter, that is, without mechanical movements or electrical signal addressing. Only one division-of-amplitude device is required, besides the two biaxial crystals, to completely characterize any state of polarization, including partially polarized and unpolarized states. In addition, a mathematical model describing the system is included. Experimental images of the intensity distribution related to different input polarization states are provided. These intensity patterns are compared with simulated values, proving the potential of polarimeters based on biaxial crystals. () 2013 Optical Society of America

OCIS codes: (120.5410) Polarimetry; (120.2130) Ellipsometry and polarimetry; (160.1190) Anisotropic optical materials; (260.1180) Crystal optics; (260.1440) Birefringence.

http://dx.doi.org/10.1364/OL.38.004100
\end{abstract}

Polarimeters are the basic devices to measure the polarization of light (Stokes polarimeters) or characterize the polarimetric properties of a polarizing sample (Mueller polarimeters) from radiometric measurements. This polarimetric information is crucial in a large number of applications, such as in medicine, to enhance the image contrast of samples [1]; in material characterization, to determine their thickness and refraction indices [2]; or in astronomy, to obtain quantitative information about stars [3].

Stokes polarimeters determine the state of polarization (SOP) of the light beam by projecting the studied incident light over a set of different polarization analyzers, known as polarization system analyzer (PSA). If the PSA includes as a minimum four linearly independent polarizing analyzers (PAs), the SOP is fully determined, constituting a complete Stokes polarimeter. A visual way to determine if the instrument is a complete polarimeter is to represent the set of PAs on the Poincaré sphere [4]. When the PAs are confined in a plane (i.e., a slide of the Poincare sphere), the resulting polarimeter is incomplete. On the contrary, if the PSA arrangement confines a certain volume in the Poincare sphere, one obtains a complete polarimeter $[\underline{5}, \underline{6}]$.

Many polarimeter architectures have been proposed in the literature [1 $-\underline{3}, \underline{5}-11]$. Roughly, punctual polarimeters can be classified depending on the acquisition method on which they are based [12]: time sequential measurements [5,7], polarization modulation [9], and division of amplitude [10]. As a consequence of the specific acquisition method applied, each category presents certain limitations in the measurement. For instance, time sequential polarimeters and polarization modulator based polarimeters require mechanical movements of polarizing elements [11] or electrical addressing to liquid crystal panels [ $[\overline{5,7}]$. Therefore, errors related to misalignments or deviations in the phase-voltage look-up table are always present. In addition, time-sequential polarimeters may present false polarization effects due to changes during data acquisition. Finally, division-of-amplitude polarimeters divide the studied beam in different subbeams, which are simultaneously projected to different polarization analyzers (in general, four of them).

In this Letter, we present a novel concept to measure states of polarization based on the mapping that the conical refraction (CR) phenomenon produces between the polarization of the input beam and the transverse intensity distribution along the light ring of the output beam [13]. This concept is adapted into the design of a new division-of-amplitude polarimeter based on two biaxial crystals and a single division-of-amplitude device. By using this alternative, not only are the benefits of amplitude division polarimeters present but also a large data redundancy is achieved, leading to a reduction of the variance in the measurements.

In CR [13-21], when a focused input Gaussian beam propagates along the optic axis of a biaxial crystal, it is transformed into a light ring, as shown in Fig. 1(a). This light ring is most sharply resolved at the focal plane. The radius of the CR ring, $R_{0}$, can be obtained from the product of the crystal's length, $L$, and its conicity, $\alpha$; that is, $R_{0}=L \alpha$. The conicity of the crystal depends on the principal refractive indices of the crystal through $\alpha=\sqrt{\left(n_{2}^{2}-n_{1}^{2}\right)\left(n_{3}^{2}-n_{2}^{2}\right)} / 4 n_{1} n_{3}$ [15]. One interesting peculiarity of the CR light ring is that it splits into two concentric bright rings separated by a dark (Poggendorff) ring under conditions of $R_{0} \gg w_{0}$, where $w_{0}$ is the waist radius of the focused input beam. Additionally, $w_{0}$ is also the width of each of these rings.
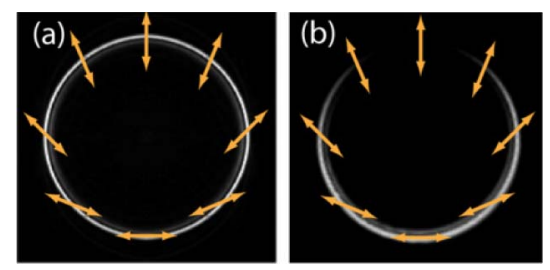

Fig. 1. CR light ring for (a) a circularly polarized input beam and (b) a horizontal linearly polarized input beam. Orange double arrows show the polarization distribution at each point of the CR ring. 
One of the most interesting features of the CR effect is the polarization distribution along the ring. Each point of the light ring is linearly polarized, with the polarization plane rotating along the ring so that every pair of diagonally opposite points has orthogonal polarizations [see orange double arrows in Fig. 1(a)]. In other words, at a certain point of the $\mathrm{CR}$ ring, given by its azimuthal angle $\varphi$, the plane of the electric field, $\Phi$, will be

$$
\Phi=\frac{\varphi+\varphi_{C}}{2},
$$

where $\varphi_{C}$ is the orientation of the plane of optic axes of the biaxial crystal [16]. Therefore, the biaxial crystal projects the input beam into an infinite number of linearly polarized states. This is the fundamental physical property on which our polarimeter is based. If the input beam is circularly polarized or unpolarized, the azimuthal intensity along the ring is constant [Fig. 1(a)]. In contrast, for linearly polarized input beams the light ring possesses a point of null intensity and a maximum intensity point placed diagonally opposite to the first one [Fig. 1(b)].

By considering all the optical features of the CR phenomenon explained just above, we propose the design of a polarimeter based on two biaxial crystals, sketched in Fig. 2. The studied light beam $\left(S_{\text {in }}\right)$, by means of a division-of-amplitude device, is split in two subbeams that are analyzed separately by two different PA arms. Both biaxial crystals were cut with one of the optic axes perpendicular to the slab faces. In both arms, lens 1 focuses the beam, which passes along the optical axis of the biaxial crystal, forming the $\mathrm{CR}$ ring at its focal plane (plane $P$ ). Lens 2 images the CR ring into the CCD camera, with a certain magnification. Thus, the intensity distribution acquired by the cameras will depend on the incident SOP $\left(S_{\text {in }}\right)$. Concerning the first arm, the obtained intensity pattern can be understood as the result of projecting the incident beam over a set of linear polarizers arranged in a circle. The orientation of the transmission axis of those polarizers is rotated (from $0^{\circ}$ to $180^{\circ}$ ) over the complete circle [see Fig. 1(a)]. In order to obtain ellipticity information, we need the second arm, which includes a quarter wave plate (QWP) before the biaxial crystal. In this way, the intensity pattern in the plane $P$

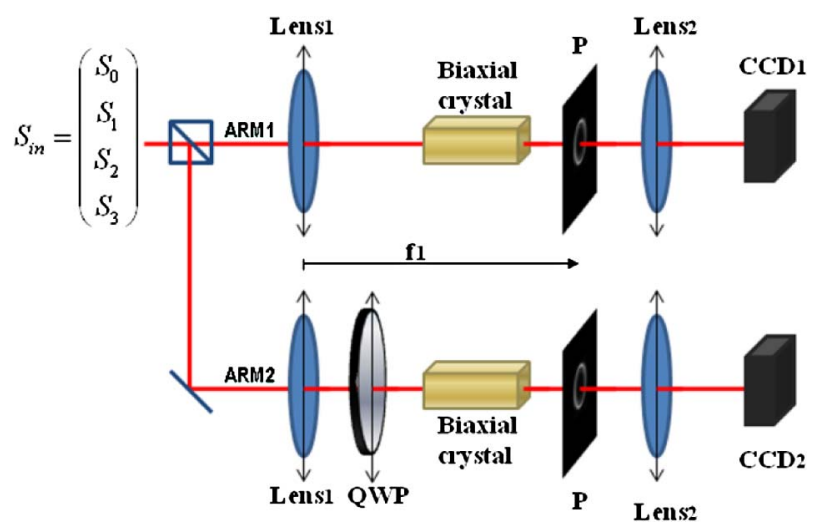

Fig. 2. Proposed setup of the polarimeter based on two biaxial crystals. The incident light beam is divided into two arms for separate analysis. for the second arm can be understood as the result of projecting the incident beam over a QWP and then over the set of rotated polarizers arranged in a circle.

By using the Mueller matrices of a QWP and a rotated linear polarizer [22], we can calculate the expressions of the PAs of both arms. These PAs will be expressed as a function of $\varphi$, the angular position at the ring. Finally, the intensity distribution measured by the two cameras will be the projection of the incident Stokes vector $\left(S_{\text {in }}\right)$ over these PAs:

$$
I_{\mathrm{CCD} 1}(\varphi)=\frac{1}{2}\left(\begin{array}{llll}
1 & \cos \varphi & \sin \varphi & 0
\end{array}\right) \cdot\left(\begin{array}{llll}
S_{0} & S_{1} & S_{2} & S_{3}
\end{array}\right)^{T},
$$

$$
I_{\mathrm{CCD} 2}(\varphi)=\frac{1}{2}\left(\begin{array}{llll}
1 & \cos \varphi & 0 & \sin \varphi
\end{array}\right) \cdot\left(\begin{array}{llll}
S_{0} & S_{1} & S_{2} & S_{3}
\end{array}\right)^{T},
$$

where we have fixed the orientation of the QWP at $0^{\circ}$ for convenience, although any other angle would have led to a complete polarimeter. In addition, these expressions can be rewritten as a function of the total intensity $\left(S_{0}\right)$, the degree of polarization (DoP) [4], the azimuth $(\alpha)$, and the ellipticity $(\varepsilon)$ of the incident SOP

$$
I_{\mathrm{CCD} 1}(\varphi)=\frac{S_{0}}{2}\left\{1+\mathrm{DoP} \cdot \cos 2 \varepsilon\left[2 \cos ^{2}\left(\frac{\varphi}{2}-\alpha\right)-1\right]\right\},
$$

$I_{\mathrm{CCD} 2}(\varphi)=\frac{S_{0}}{2}+\frac{\mathrm{DoP} \cdot S_{0}}{2}[\cos 2 \varepsilon \cos 2 \alpha \cos \varphi+\sin 2 \varepsilon \sin \varphi]$.

Note that Eq. (4) describes the intensity distribution along the ring due to the $\mathrm{CR}$ phenomenon for any input SOP. This equation is a generalization of the equations presented in [16], describing the particular cases of linearly and circularly fully polarized states.

The PAs of both arms are plotted upon the Poincaré sphere in Fig. 3. If the orientation of the QWP is rotated an angle $\theta$, the curve of the PAs represented upon the Poincaré sphere corresponding to arm 2 will be rotated $2 \theta$ over the S3 axis. Note that by using only a single arm, the PAs draw a plane in the Poincaré sphere, and consequently, they constitute an incomplete polarimeter. In particular, the PAs from arm 1 (red line in Fig. 3) do not have information about S3, and the ones from arm 2 (blue line) do not measure the S2 component. However, when we consider the whole system, the polarimeter is complete because the PAs represented upon the sphere are enclosing a certain volume.

In general, different quality indicators are used to evaluate the propagation of noise to the measurement in polarimeters, as, for instance, the condition number $(\mathrm{CN})$ $[\underline{23}]$ or the equally weighted variance (EWV) $[\underline{5}, \underline{6}]$ indicators. The $\mathrm{CN}$ (a metric widely used in polarimeter design) calculated for the arrangement of our PAs is equal to 2.00 , a value very close to the theoretical minimum of 


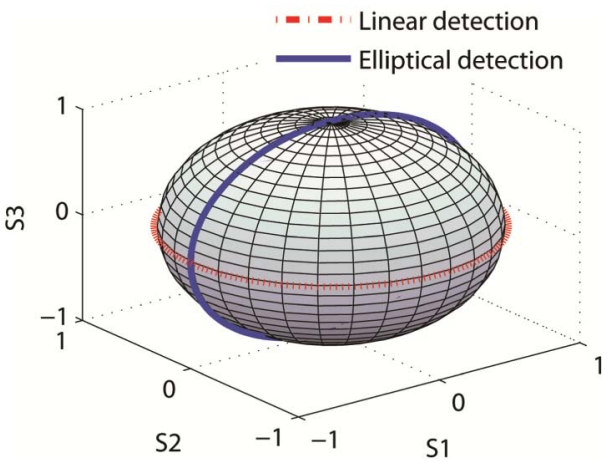

Fig. 3. PAs represented upon the Poincaré sphere. The red line corresponds to the linear detection arm and the blue line to the elliptical detection arm (assuming that the QWP is at $0^{\circ}$ ).

1.73 for polarimeters. The EWV indicator is also calculated for our proposed configuration. From simulations, we observe that the EWV value depends on the number of PAs used in the Stokes measurement [5]: the larger the number of PAs used, the smaller the EWV value obtained. For the case of 360 PAs per arm, that is, 720 in total, the $\mathrm{EWV}$ is 0.0153 . This value is much smaller than the one reported in [5] for 100 PAs (0.1).

To show and prove the measurement principle of the polarimeter design proposed above, we have experimentally implemented the two arms of Fig. $\underline{2}$ and analyzed different input SOPs. In the experimental implementation, the input light is obtained from a $640 \mathrm{~nm}$ diode laser coupled to a monomode fiber. The two biaxial crystals used in the setup were cut from a monoclinic centrosymmetric $\mathrm{KGd}\left(\mathrm{WO}_{4}\right)_{2}$ crystal. Their polished entrances (cross section $6 \mathrm{~mm} \times 4 \mathrm{~mm}$ ) have parallelism with less than 10 arc sec, and they are perpendicular to one of the two optic crystal axes within a $1.5 \mathrm{mrad}$ misalignment angle. Their lengths, $L=23.38 \mathrm{~mm}$ (measured with precision of less than $100 \mathrm{~nm}$ ), and their conicity, $\alpha=17 \mathrm{mrad}$, provide a $\mathrm{CR}$ ring of radius $R_{0}=397 \mu \mathrm{m}$.

Figure $\underline{4}$ shows the two experimental images acquired by the two cameras when seven particular SOPs illuminate the system. The used SOPs are linearly polarized light at $0^{\circ}, 90^{\circ}, 45^{\circ}$, and $135^{\circ}$, right- and left-handed circularly polarized light, and unpolarized light. The first row of Fig. 4 corresponds to the first camera, that is, projecting over linear polarization analyzers. Thus, when this camera analyzes a linear SOP [Figs. $\underline{4(a)-4(d)}$ ], the intensity patterns consist of broken rings with a

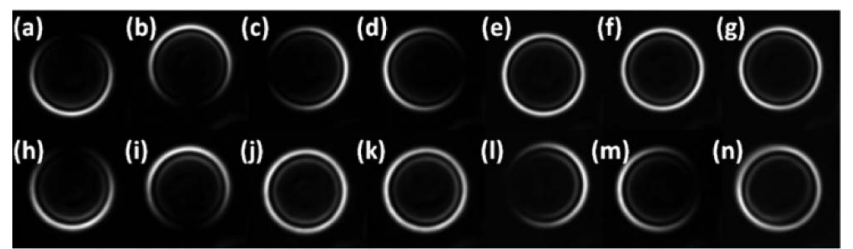

Fig. 4. Experimental images acquired by cameras of (a)-(g) arm 1, and (h)-(n) arm 2 when illuminating with linearly polarized light at (a) and (h) $0^{\circ}$, at (b) and (i) $90^{\circ}$, at (c) and (j) $45^{\circ}$, at (d) and (k) $135^{\circ}$, with circularly polarized light (e) and (l) right-handed and (f) and (m) left-handed and with (g) and (n) unpolarized light. maximum and a null of intensity in diametrically opposite positions in the ring. This broken ring rotates as we rotate the azimuth of the analyzed SOP, as it is described in Eq. (4). As explained above, circularly polarized light and unpolarized light [Figs. $4(\mathrm{e})-4(\mathrm{~g})]$ result in a ring of constant intensity. In the second row of Fig. 4 , the intensity distributions differ from the first row because of the presence of the QWP. When illuminating with $0^{\circ}$ or $90^{\circ}$ linear SOPs, the polarization is not modified by the QWP (oriented at $0^{\circ}$ ), and for this reason, the intensity distributions [Figs. 4(h) and 4(i)] are identical to the ones acquired by the first camera. In addition, unpolarized light remains unpolarized after the QWP, so that a uniform ring is visualized in Fig. 4(n). Finally, when projecting the linear SOPs at $45^{\circ}, 135^{\circ}$ and right-handed and left-handed circular SOPs over the QWP, they are respectively transformed to left-handed and right-handed circular SOPs and linear SOPs at $45^{\circ}$ and $135^{\circ}$. Then, by taking into account the actual SOP impinging the biaxial crystal, and considering the effect of the crystal over these SOPs as explained above, we understand the intensity distribution of Figs. $4(\mathrm{j})-4(\mathrm{~m})$.

By analyzing the data from these experimental images, we have extracted the intensity profile along the rings, plotted in red points in Fig. 5. Moreover, we have included in a blue continuous line the simulated intensity profiles by using Eqs. (2) and (3). We observe good agreement between experimental and simulated intensity profiles.

Note that if only the linear arm is used, there is not distinction between unpolarized light and right and left circularly polarized light [see Figs. 5(e)-5(g)]. In the same way, the elliptical arm cannot distinguish between linearly polarized light at $45^{\circ}, 135^{\circ}$, and unpolarized light [see Figs. 5(j), 5(k), and 5(n)]. Nevertheless, by using the two intensity profiles corresponding to both arms, we are able to distinguish each SOP, including unpolarized light beams.

Thus, the results given in Figs. 4 and 5 constitute an experimental proof of the capability of the presented design to perform polarimetric measurements, as the whole polarimetric content of any input SOP can be determined. In fact, by applying a data reduction procedure

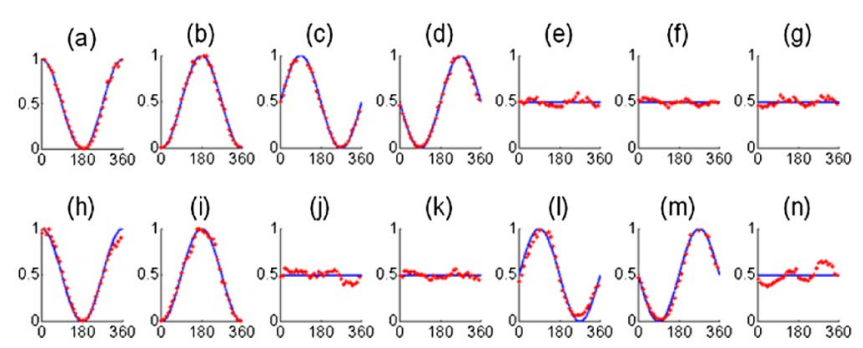

Fig. 5. Simulated intensity profile in the continuous blue line and experimental intensity profile in the red spots, as a function of $\varphi$, the position along the ring of (a)-(g) arm 1 and (h)-(n) arm 2 ; when illuminating with linearly polarized light at (a) and (h) $0^{\circ}$, at (b) and (i) $90^{\circ}$, at (c) and (j) $45^{\circ}$, and at (d) and (k) $135^{\circ}$, with circularly polarized light (e) and (l) right-handed and (f) and (m) left-handed and with (g) and (n) unpolarized light. 
[14] to the two intensity profiles obtained (arm 1 and 2), a quantitative estimation of any input SOP can be achieved.

In conclusion, this work presents a new concept for Stokes vector metrology by means of analyzing the characteristic intensity pattern associated with the CR phenomenon occurring in biaxial crystals. This idea is developed by proposing the design of a division-ofamplitude complete and punctual Stokes polarimeter based on two biaxial crystals. The setup requires two cameras to acquire the intensity distribution along the $\mathrm{CR}$ light rings. Although other punctual polarimeters only require one radiometer $[5,8]$, this drawback is compensated by the strengths of the biaxial crystal based polarimeter, namely no electrical addressing is needed [asliquid crystal based polarimeters], eliminating systematic errors related to the liquid crystal calibration; static measurements (no moving elements), avoiding misalignment errors; quasi-real-time measurements (only limited by the acquisition and computational time); large data redundancy, leading to a significant reduction of the variance; and ability to characterize any SOP, including partially polarized or unpolarized light. All these features make the instrument very useful for practical applications in polarimetry.

However, since the CR phenomenon is very sensitive to the incidence angle, the necessity of performing a very accurate alignment is expected. Additionally, an experimental calibration of the PAs is strongly recommended in order to take into account possible polarization defects of the elements in the setup [12]. For instance, some differences with the theoretical configuration may be introduced in the experimental implementation due to polarization effects related to the division-of-amplitude device used in the setup, or due to experimental deviations of the QWP retardance value. In a future study, we will provide a thorough description of the implementation, calibration, and measurement analysis related to a particular polarimeter architecture including two biaxial crystals. In addition, it could be very interesting to extend this proposal to any wavelength where the biaxial crystals are transparent, expanding the range of applicability of the discussed polarimeter to spectral regions such as the infrared or ultraviolet.

We acknowledge financial support from the Spanish MINECO (FIS2012-39158-C02-01, FIS2011-23719, BES2010-031696, and AP2010-2310) and the Catalan
Government (SGR2009-00347). We would like to thank Dr. Todor Kirilov for providing us the biaxial crystals.

\section{References}

1. A. Pierangelo, A. Benali, M. R. Antonelli, T. Novikova, P. Validire, B. Gayet, and A. De Martino, Opt. Express 19, 1582 (2011).

2. A. Lizana, M. Foldyna, M. Stchakovsky, B. Georges, D. Nicolas, and E. Garcia-Caurel, J. Phys. D 46, 105501 (2013).

3. N. Uribe-Patarroyo, A. Alvarez-Herrero, R. L. Heredero, J. C. del Toro Iniesta, A. C. López Jiménez, V. Domingo, J. L. Gasent, L. Jochum, and V. Martínez Pillet, Phys. Status Solidi C 5, 1041 (2008).

4. D. H. Goldstein, Polarized Light, 3rd ed. (CRC Press, 2010).

5. A. Peinado, A. Lizana, J. Vidal, C. Iemmi, and J. Campos, Opt. Express 18, 9815 (2010).

6. D. S. Sabatke, M. R. Descour, E. L. Dereniak, W. C. Sweatt, S. A. Kemme, and G. S. Phipps, Opt. Lett. 25, 802 (2000).

7. E. Garcia-Caurel, A. De Martino, and B. Drévillon, Thin Solid Films 455-456, 120 (2004).

8. D. H. Goldstein, Appl. Opt. 31, 6676 (1992).

9. O. Arteaga, J. Freudenthal, B. Wang, and B. Kahr, Appl. Opt. 51, 6805 (2012).

10. E. Compain and B. Drevillon, Appl. Opt. 37, 5938 (1998).

11. D. H. Goldstein and R. A. Chipman, J. Opt. Soc. Am. A 7, 693 (1990).

12. R. A. Chipman, in Handbook of Optics, 2nd ed. (McGrawHill, 1995).

13. M. V. Berry and M. R. Jeffrey, Prog. Opt. 50, 13 (2007).

14. A. M. Belskii and A. P. Khapalyuk, Opt. Spectrosc. 44, 436 (1978).

15. T. K. Kalkandjiev and M. A. Bursukova, Proc. SPIE 6994, 69940B (2008).

16. A. Turpin, Y. V. Loiko, T. K. Kalkandjiev, and J. Mompart, Opt. Lett. 38, 1455 (2013).

17. A. Turpin, Y. Loiko, T. K. Kalkandjiev, and J. Mompart, Opt. Lett. 37, 4197 (2012).

18. A. Abdolvand, K. G. Wilcox, T. K. Kalkandjiev, and E. U. Rafailov, Opt. Express 18, 2753 (2010).

19. D. P. O'Dwyer, C. F. Phelan, K. E. Ballantine, Y. P. Rakovich, J. G. Lunney, and J. F. Donegan, Opt. Express 18, 27319 (2010).

20. M. V. Berry, M. R. Jeffrey, and J. G. Lunney, Proc. R. Soc. London, Ser. A 462, 1629 (2006).

21. Y. Mikhailichenko, Russ. Phys. J. 50, 788 (2007).

22. D. H. Goldstein, Polarized Light, 2nd ed. (Marcel Dekker, 2003).

23. P. Taylor, Theory and Applications of Numerical Analysis, 2nd ed. (Academic, 1996). 